\title{
EvAAL'12 awards
}

\section{Introduction}

"Evaluating Ambient Assisted Living Systems through Competitive Benchmarking" (EvAAL) is an annual international competition that addresses the "grand" challenge of evaluation and comparison of Ambient Assisted Living (AAL) systems and platforms, with the final goal to assess the autonomy, independent living and quality of life that AAL systems may grant to their end users. The EvAAL event is a live competition and this year edition is focused on two pillars of AAL: Indoor localization and activity recognition.

\subsection{Indoor localization track competitors}

Seven teams were accepted to the indoor localization competition, namely CAR (from the Centre for Automation and Robotics, Spain) [1], LOCOSmotion (from the University of Duisburg-Essen, Germany) [2], OwlPS (from the Institute Femto-st, France) [3], CPS Group @ Utah (from the University of Utah, USA) [4], TAIS (from the University of Sevilla, Spain) [5], iLocPlus (from Stuttgart University of Applied Sciences and iHomeLab at Lucerne University of Applied Sciences) [6], and Smart-Condo (from the University of Alberta, Canada) [7]. All of them are described in EvAAL website: evaal.aaloa.org.

\subsection{Activity recognition track competitors}

After peer review, five teams were accepted but one of them withdrew due to financial cutbacks of its institution. Hence only four competitors participated in the challenge, CUJ (from the University of Chiba, Japan) [8], CMU (from Carnegie Mellon and Utah Universities, USA) [9], DCU (from Dublin City University, Ireland) [10] and USS (from University of Seville, Spain) [11]. All of them are described in EvAAL website: evaal.aaloa.org

\section{Awards}

During the 2nd edition of the International Workshop of EvAAL held in conjunction with the AAL Forum in Eindhoven (Netherlands) last September, the

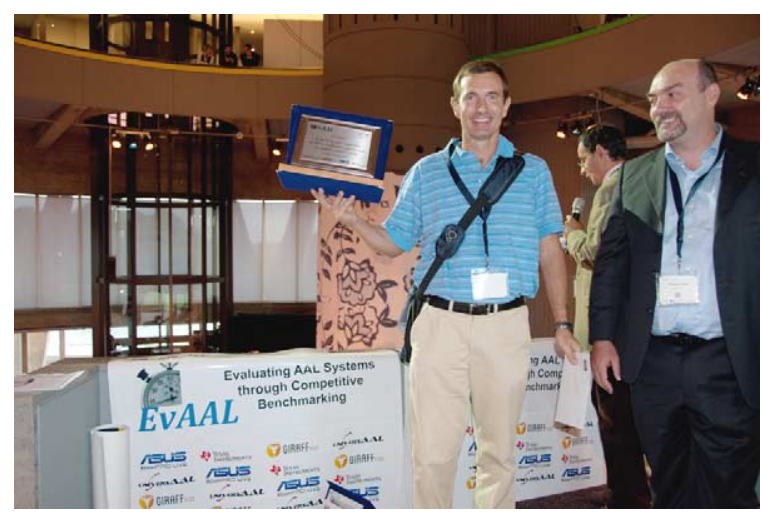

Fig. 1. Antonio Ramón Jiménez showing the first prize of Indoor Localization track.

organizers rewarded the winners of the competitions in Indoor Localization and Activity Recognition. Prizes were supported by ASUS, Texas Instrument, GiraffPlus and UniversAAL.

The prize for the best solution in both tracks consists of a plaque and a robot from Texas Instrument. Second prize consists of a plaque and a ASUS Xtion and third prize is a plaque.

The chairs of EvAAL assigned the awards based on the results of each competition, that were assessed by the Evaluation Committees.

\subsection{Indoor localization track}

CAR Team from the Centre for Automation and Robotics from CSIC (Spain) won the first prize. The photo in Fig. 1 shows Francesco Furfari (future General Chair in EvAAL'13) presenting a certificate to competitor Antonio Ramón Jiménez Ruiz from CAR Team. The second prize for this track was awarded to CPS Group @ Utah from the University of Utah (USA). The photo in Fig. 2 shows Juan Antonio Álvarez (Chair of Activity Recognition track), Stefano Chessa (General Chair of EvAAL'12) and Maurizio Bocca from CPS Group. Third prize was awarded to OwlPS Team from Institute Femto-st (France). The photo in Fig. 3 shows Paolo Barsocchi (Chair of Indoor Localization track) congratulating Philippe Canalda from OwlPS Team. 


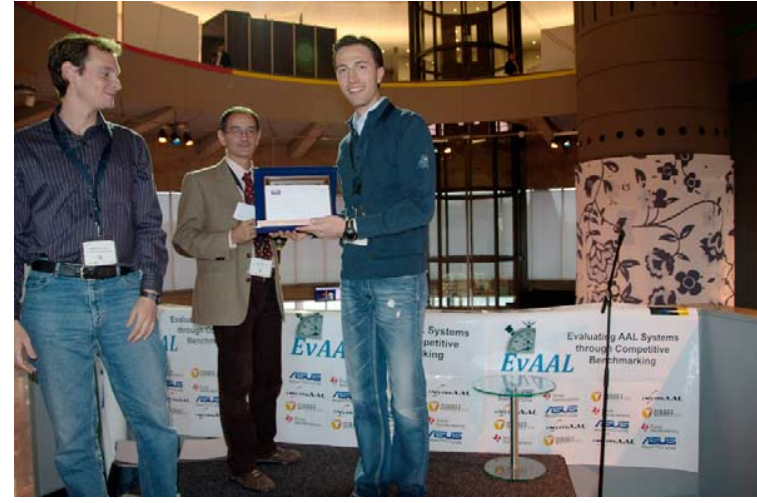

Fig. 2. Maurizio Bocca showing the second prize of Indoor Localization track.

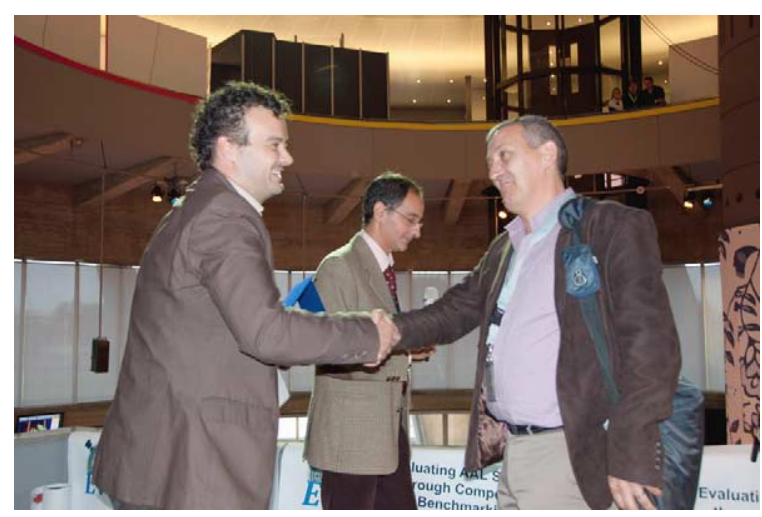

Fig. 3. Philippe Canalda (right) being congratulated by Paolo Barsocchi.

\subsection{Activity recognition track}

Ameva Team from the University of Seville (Spain) won the first prize in this track. The photo in Fig. 4 shows Francesco Furfari giving the prize to Luis Miguel Soria from Ameva Team.

The second prize was awarded to Utah-CMU team from the University of Utah and Carnegie Mellon University. Maurizio Bocca as representative of the team received the award. Third prize was awarded to Chiba Team from the University of Chiba (Japan). The photo in Fig. 5 shows José González showing his prize close to Stefano Chessa.

\section{References}

[1] A.R. Jiménez Ruiz, F. Seco Granja, J.C. Prieto Honorato, and J.I. Guevara Rosas, Accurate pedestrian indoor navigation by tightly coupling foot-mounted imu and rfid measurements, IEEE T. Instrumentation and Measurement 61(1) (2012), 178189.

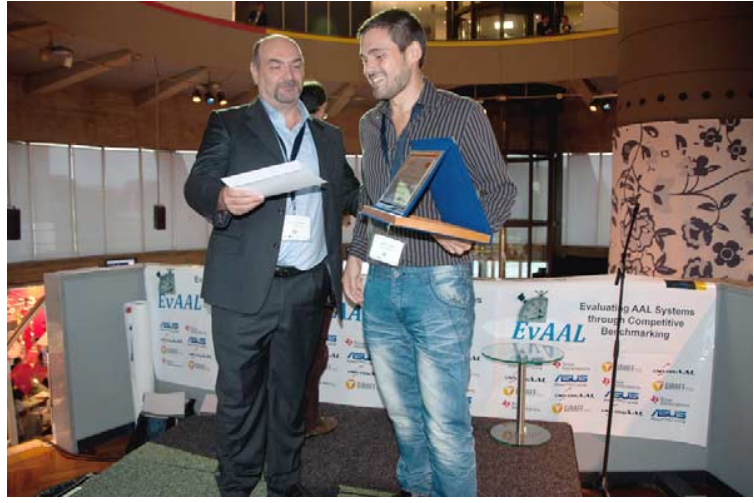

Fig. 4. Luis Miguel Soria receiving the first prize.

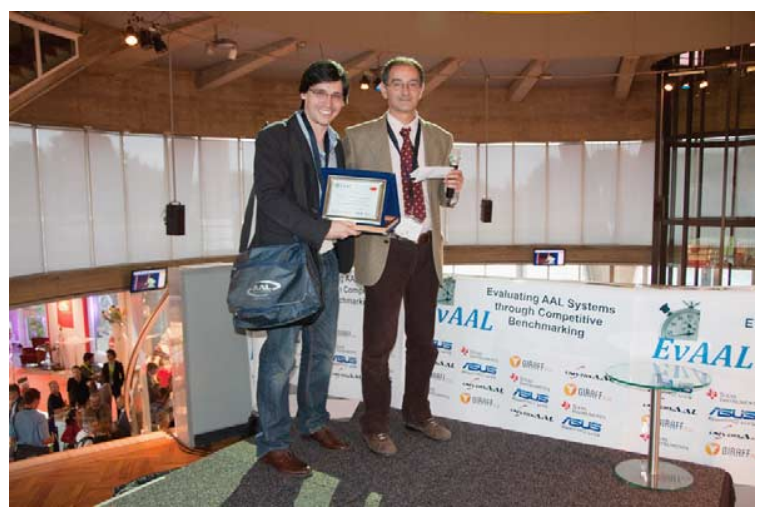

Fig. 5. José González showing the third prize.

[2] S. Wagner, M. Handte, M.A.Z. Zamalloa, and P.J. Marrón, On optimal tag placement for indoor localization, in: PerCom, 2012, pp. 162-170.

[3] M. Cypriani, P. Canalda, and F. Spies, Owlps: A self-calibarted fingerpringing-based Wi-Fi positioning system, Communications in Computer and Information Science 309 (2012), 36-51.

[4] J. Wilson and N. Patwari, See-through walls: Motion tracking using variance-based radio tomography networks, IEEE Trans. Mob. Comput. 10(5) (2011), 612-621.

[5] A.V. Medina, J.A. Gómez, O. Rivera, E. Dorronzoro, and M. Merino, Fingerprint indoor position system based on openmac, in: WINSYS, 2011, pp. 47-52.

[6] S. Knauth, L. Kaufmann, R.K. Christian Jost, and A. Klapproth, The iloc ultrasound indoor localization system at the EvAAL 2011 competition, Communications in Computer and Information Science 309 (2012), 52-64.

[7] N.M. Boers, D. Chodos, J. Huang, P. Gburzynski, I. Nikolaidis, and E. Stroulia, The smart condo: Visualizing independent living environments in a virtual world, in: PervasiveHealth, 2009, pp. $1-8$.

[8] M. Nergui, Y. Yoshida, J. Gonzalez, Y. Koike, M. Sekine, and Y. Wenwei, Human motion tracking and measurement by a mobile robot, in: The International Conference on Intelligent Unmanned Systems 2011 - ICIUS2011, 2011. 
[9] J.-H. Hong, J. Ramos, and A.K. Dey, Understanding physiological responses to stressors during physical activity, in: Proc. of the 2012 ACM Conference on Ubiquitous Computing, ser. UbiComp '12, ACM, New York, NY, USA, 2012, pp. 270-279. [Online]. Available: http://doi.acm.org/ $10.1145 / 2370216.2370260$
[10] N. Li, M. Crane, and H.J. Ruskin, Automatically detecting "significant events" on sensecam, Ercim News 87 (2011), 3031

[11] L.G. Abril, F.J. Cuberos, F. Velasco, and J.A. Ortega, Ameva: An autonomous discretization algorithm, Expert Syst. Appl. 36(3) (2009), 5327-5332. [Online]. Available: http://dblp. uni-trier.de/db/journals/eswa/eswa36.html\#AbrilCVO09. 